\title{
THE EFFECT OF THE ADOLESCENT GROWTH SPURT ON EARLY POSTERIOR SPINAL FUSION IN INFANTILE AND JUVENILE IDIOPATHIC SCOLIOSIS
}

\author{
FRITZ L. HEFTI, MICHAEL J. MCMASTER
}

\author{
From the Edinburgh Scoliosis Unit
}

\begin{abstract}
Twenty-four children with infantile or juvenile idiopathic scoliosis had their spines corrected and solidly fused posteriorly before the age of eleven years. The growth of the fusion area was then accurately measured for a mean of 4.5 years during the adolescent growth spurt. During this period all longitudinal growth in the posterior elements ceased. The vertebral bodies continued to grow anteriorly, but the thick posterior fusion prevented the development of a lordosis. Initially the anterior growth was accommodated by narrowing of the intervertebral disc spaces, but eventually the vertebral bodies bulged laterally towards the convexity and pivoted on the posterior fusion, giving rise to loss of correction, increasing vertebral rotation and recurrence of the rib hump.
\end{abstract}

Posterior spinal fusion with Harrington instrumentation is a widely accepted method of correcting and preventing increasing deformity in growing patients with scoliosis. Risser et al. (1966) stated that due to the biologic plasticity of bone, the posterior fusion was elongated by the tension stress of continued growth of the unfused vertebral bodies anteriorly. It is, however, more widely accepted that posterior fusion stops all longitudinal growth in the posterior elements and may therefore cause a relative shortening of the spine as the child grows to maturity (Winter 1971). Ponseti and Friedman (1950) also suggested that an increasing lordosis may develop in the fused area due to the continued growth of the vertebral bodies and the lack of growth in the posterior elements. Tanner, Whitehouse and Takaishi (1966) showed that the longitudinal rate of growth of the spine is not constant: there is a period of accelerated growth which occurs in British girls between the ages of 10.5 and 13.5 years, and in boys between 12.5 and 15.5 years. Little is known of the effect of this adolescent growth spurt on the spines of children which have been solidly fused posteriorly before its onset. Progressive infantile and juvenile idiopathic curves commence before the age of 10 years and may require an early posterior fusion to control their progression. In the few previous studies of

Fritz L. Hefti, MD, Oberarzt

Orthopaedische Universitătsklinik, Kinderspital, 4058 Basel, Switzerland.

Michael J. McMaster MD, FRCS, Consultant Orthopaedic Surgeon Princess Margaret Rose Orthopaedic Hospital, Edinburgh EH10 7ED, Scotland.

Requests for reprints to be sent to Mr M. J. McMaster.

(C) 1983 British Editorial Society of Bone and Joint Surgery

$0301-620 \mathrm{X} / 83 / 3086-0247 \$ 2.00$ early posterior spinal fusion for scoliosis the large majority of patients had congenital scoliosis, and there were relatively few with progressive infantile or juvenile idiopathic curves (Moe, Sundberg and Gustilo 1964; Winter 1971; Winter and Moe 1982; Letts and Bobechko 1974). In congenital scoliosis the longitudinal growth of the spine is already impaired both anteriorly and posteriorly by the congenital unilateral failure of formation or segmentation of the vertebrae. In idiopathic scoliosis vertebral growth is relatively unimpaired and the effect of posterior fusion before the adolescent growth spurt may be very different.

Posterior spinal fusion had been recommended at the age of 10 years in children with progressive infantile and juvenile idiopathic scoliosis, to control the more rapid deterioration which usually occurs during the adolescent growth spurt and cannot be adequately controlled by bracing (James 1967). A previous study by one of us of 12 children with progressive infantile idiopathic curves which were solidly fused at ten years of age showed that after a mean of seven years they had lost a mean of 17 degrees of correction before becoming stable (McMaster and Macnicol 1979). Nearly all of this loss occurred in the first few years, and was due to bending of the solid but plastic fusion mass during the adolescent growth spurt. Harrington instrumentation was not used in these children and was seldom used in the few other reports of early posterior spinal fusion for idiopathic scoliosis which also recorded a relatively high loss of correction (Moe et al. 1964; Winter 1971; Letts and Bobechko 1974).

This paper is concerned with early posterior fusion of progressive infantile and juvenile idiopathic scoliosis, and its purpose is twofold. Firstly to report a prospective study by the senior author of the effect of Harrington 
instrumentation and a massive onlay of autogenous iliac bone grafts in an attempt to produce a strong thick posterior fusion which would resist bending during the adolescent growth spurt. Secondly to detect any adverse effects such as relative stunting of growth, increasing lordosis or rotational deformities of the spine which might result from early posterior fusion in these patients.

\section{CLINICAL MATERIAL}

At the Princess Margaret Rose Orthopaedic Hospital, Edinburgh, during the period May 1975 to April 1980, 24 patients with infantile or juvenile idiopathic scoliosis underwent posterior spinal fusion with Harrington instrumentation before the age of 11 years. All of these operations were performed by one of us (M. McM), using the same technique of correction and fusion. Twentythree patients were recalled and reviewed personally by both authors after an average of 4.5 years (range 2 years to 7 years and 2 months) after operation. Of the 23 patients reviewed, 15 had progressive infantile idiopathic scoliosis (age of onset between 3 months and 3 years) and eight had juvenile idiopathic scoliosis (age of onset between 8 years and 9 years 6 months). There were 14 boys and nine girls. The mean age at first presentation to the scoliosis clinic was 5.5 years (range one year and 6 months to 10 years and 6 months), and all except two patients were initially treated in a Milwaukee brace for an average of 4.5 years (range one to 8 years) before operation. The mean age at operation was 10 years and one month (range 8 years and 6 months to 11 years). Twenty-two patients had single thoracic curves; one had a thoraco-lumbar curve. Thirteen curves were to the left and 10 to the right.

\section{METHODS}

Operative technique

The scoliosis was corrected by the Harrington distraction system with the hook inserted into the neutral vertebra at either end of the curve. A single distraction rod was used without the compression system (Harrington 1962). There was no pre-operative traction nor the use of corrective plaster jackets. The spine was fused posteriorly from the upper to the lower Harrington hooks by the interfacetal, intertransverse fusion technique described by Moe (1958). There was then a deep and thorough decortication of all the posterior bony structures from the midline out to the tips of the transverse processes. This was followed by the application throughout the fusion area of large amounts of fresh autogenous iliac bone which had been cut into matchsticks. On average 11 vertebrae were fused (range 10 to 15 vertebrae). Figure 1 shows the apex of the curves, the side of the convexity and the vertebrae fused.

\section{Post-operative care}

After the operation all the patients were nursed free in bed for 7 to 10 days. An underarm plaster jacket was then applied with the patient lying on the spinal table but without traction. The jacket was carefully moulded over the iliac crests and rib hump to support the curve. The patients were then immediately mobilised and allowed home within a few days. They returned to normal school and activities within a few weeks but were not allowed to participate in contact sports.

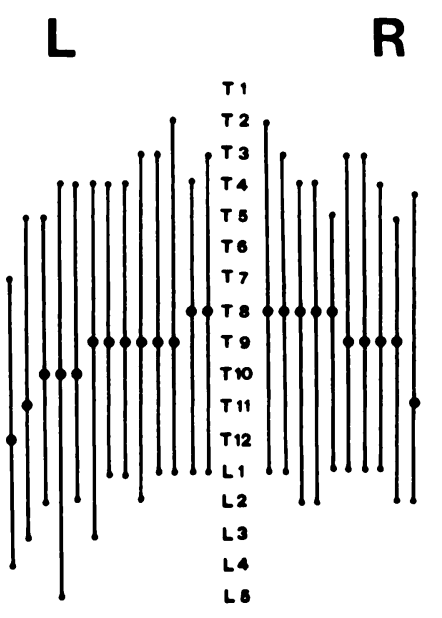

Fig. 1

Apex of curves, side of convexity and vertebrae fused.

According to a policy in earlier years, but now discontinued, 11 patients had the spine explored surgically after six months and all were found to have a solid fusion. After ten months the underarm jacket was removed and all patients were allowed unrestricted activities without external support.

\section{Spinal assessment}

The patients were routinely reviewed and radiographed when the plaster jacket was removed, then at one year, at 18 months and at 27 months after the operation. Thereafter they were seen at yearly intervals until the iliac apophyses were fully formed and fused and the spine was considered to be skeletally mature (Zaousis and James 1958).

All of the patients were specially recalled for the purpose of this investigation. Their spines were examined and the standing and sitting heights were recorded. Clinical photographs were taken and compared with those previously taken before operation and one year after operation. Antero-posterior and lateral radiographs of the erect spine were taken with the tube centred over the middle of the fused area.

\section{Radiographic measurements of the fused region}

The severity of the scoliosis was measured by the Cobb method on the erect antero-posterior spinal radiographs taken before operation, after the plaster jacket had been applied 7 to 10 days after operation, when the plaster jacket was removed 10 months after operation, and at 
the final follow-up. Care was taken always to measure the Cobb angles from the same levels at either end of the fused region in each patient. Serial measurements of the fused region in each patient were made as follows. First, the length of the fusion in the longitudinal axis from the mid-point on the upper surface of the vertebra at the upper end of the fusion to the mid-point on the lower surface of the vertebra at the lower end of the fusion

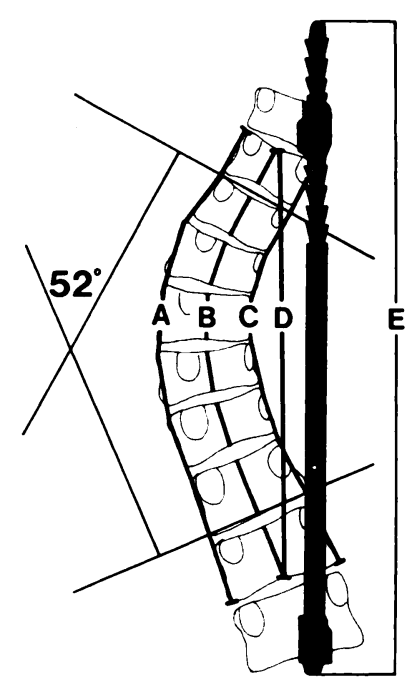

Fig. 2

\begin{abstract}
Measurements on the antero-posterior radiographs. A: Distance along the convex side. B: Sum of the lengths of the vertebral bodies in their midportion. C: Distance along the concave side. D: Length in the longitudinal axis. E: Length of the Harrington rod (= reference distance).
\end{abstract}

(distance D in Fig. 2) was measured. Secondly, the distances along the convex (distance $A$ in Fig. 2) and concave (distance $\mathrm{C}$ in Fig. 2) borders of the vertebrae in the fusion region were measured. In addition, the sum of the lengths of the vertebral bodies in their mid portion (distance B in Fig. 2) was calculated in the fused region. In order to overcome errors due to the differing magnifications of the serial radiographs, all the measurements were calibrated against the length of the Harrington rod (distance $\mathrm{E}$ in Fig. 2), which served as a reference distance for each patient. The radiographic difference in the length of the Harrington rod in each of the serial radiographs with reference to the first post-operative radiograph was used to calculate the degree of magnification, and the corresponding corrections were made to the distances measured on each radiograph. Errors due to magnification could be as large as 9 per cent, especially when comparing radiographs in plaster and without. We also compared the length of the fused region in the longitudinal axis (distance D in Fig. 2) on the postoperative radiograph with the same measurement on the pre-operative radiograph in order to estimate the increase in height produced by correcting the scoliosis. This measurement could not be corrected for magnification because of the absence of the Harrington rod on the preoperative radiograph.

Changes in rotation were measured with reference to the apical vertebral body on the serial radiographs for each patient. The distance between the border of the

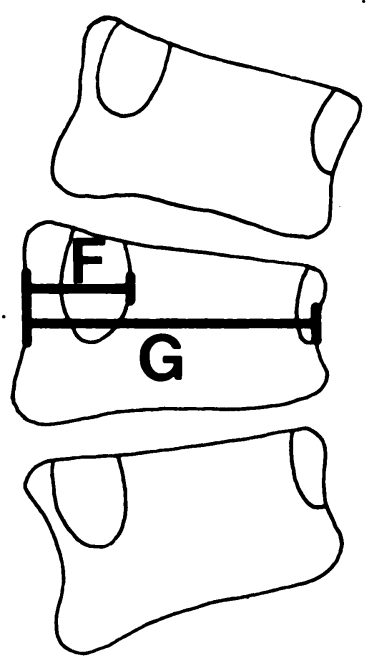

Fig. 3

Measurements of rotation. $F$ : Distance between the border of the body on the convex side and the medial border of the pedicle on the same side. G: Width of the vertebral body at its narrowest site. The ratio $F: G$ is calculated. On serial radiographs changes of this ratio on the same vertebral body result from changes in rotation.

body on the convex side and the medial border of the pedicle on the same side (distance F in Fig. 3) was measured. This was compared to the total width of the vertebral body at its narrowest site (distance $G$ in Fig. 3) and the ratio $F: G$ calculated. This ratio overcame errors in magnification and could be used for direct comparison on the serial radiographs.

\section{RESULTS}

There were no post-operative complications and all the patients developed a solid fusion. In 11 of the earlier patients the solid fusion was seen at routine surgical exploration performed six months after the fusion operation. This procedure was later discontinued because no pseudarthroses were found (McMaster 1980). Solid fusion was demonstrated on the antero-posterior, lateral and oblique radiographs of all of the patients taken when the plaster jacket was removed at 10 months.

\section{Severity of scoliosis}

Figure 4 shows the mean severity of the scoliotic curves and their range of values before operation, after appli- 
cation of the plaster jacket seven to ten days later, at 10 months when the plaster jacket was removed, and at final follow-up. It should be noted that we measured the post-operative degree of severity on the erect spinal radiograph taken after the plaster jacket was applied. Occasionally there was a very slight loss of correction between the time of insertion of the Harrington rod and the application of the plaster jacket. We believe that the radiograph taken after the plaster jacket was applied was a more realistic measurement of the degree of correction

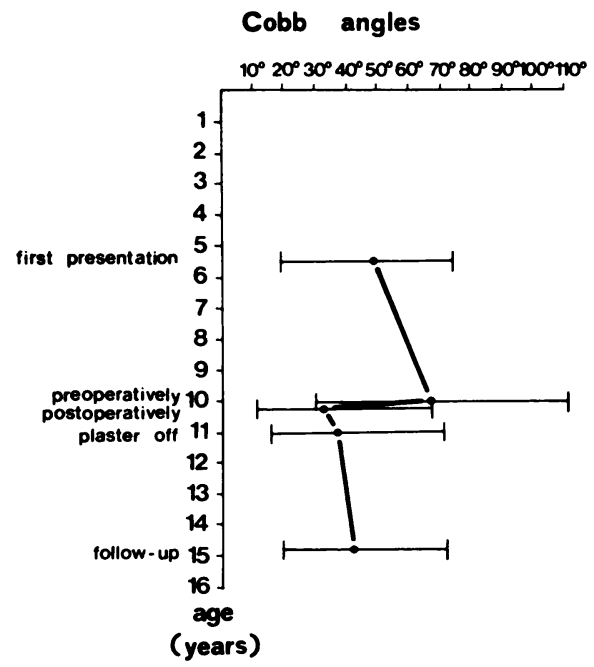

Fig. 4

Mean severity of the scoliotic curves and their range of values at first presentation, pre-operatively, seven days after operation (when plaster jacket was applied), 10 months after operation (when plaster was removed) and at final followup.

and a more suitable reference radiograph for the calculation of future loss of correction. The mean preoperative curve was 66.9 degrees (range 30 to 112 degrees) and after operation this was reduced to a mean of 32.9 degrees (range 11 to 68 degrees). The resulting correction was 50.8 per cent or 34.0 degrees (range 11 to 54 degrees). Ten months after operation and out of plaster, the mean curvature was 37.0 degrees (range 16 to 72 degrees), and there was a mean loss of correction of 4.1 degrees or 6.1 per cent. At final follow-up, a mean 4.5 years after operation, the mean scoliosis was 41.7 degrees (range 20 to 72 degrees). The total loss of correction was now 8.8 degrees (range 2 to 19 degrees) or 13.2 per cent.

\section{Severity of kyphosis}

Fifteen patients had pre-operative lateral spinal radiographs and the mean degree of kyphosis in the area of the planned fusion was 28.7 degrees (range 10 to 60 degrees). This angle was not the same as the thoracic kyphosis: it was usually smaller because we measured only the vertebrae within the fusion area and not the whole of the thoracic spine. Five patients with infantile idiopathic scoliosis had a true kyphoscoliosis with an increased kyphosis of over 40 degrees (range 44 to 64 degrees), within the planned fusion area. No patient had a preoperative true thoracic lordosis. Ten months after operation and out of plaster, the mean degree of kyphosis for all the patients was 18.4 degrees (range 10 to 33 degrees) in the fusion area. There was, therefore, a mean reduction in the pre-operative kyphosis of 10.3 degrees (range 2 to 24 degrees) or 35.9 per cent. At final followup, a mean of 4.5 years after operation, the mean kyphosis was 18.1 degrees for all the patients. In five patients there had been no change, 12 patients had a reduction in the kyphosis of up to 10 degrees but were not lordotic, and six patients had an increase in kyphosis of between 5 and 24 degrees.

\section{Longitudinal growth of the fusion area}

The mean length of the fusion area in the longitudinal axis of the post-operative radiograph (distance D in Fig. 2) was $17.4 \mathrm{~cm}$ (range 12.9 to $21.6 \mathrm{~cm}$ ). Ten months after operation there was a mean decrease in this distance, corrected for magnification, of $0.2 \mathrm{~cm}$ (range 0 to $0.6 \mathrm{~cm}$ ) and by final follow-up this distance had increased only very slightly to a mean of $0.6 \mathrm{~cm}$ (range 0.1 to $1.5 \mathrm{~cm}$ ). The mean longitudinal length of the fusion area was, therefore, $0.4 \mathrm{~cm}$ longer than that noted immediately after operation.

It was not possible to measure very accurately the increase in the longitudinal length of the fusion area before and after operation because the absence of a Harrington rod on the pre-operative radiograph prevented an assessment of the degree of magnification. An estimation of the difference in magnification on the postoperative radiograph was approximately 10 per cent, and with this correction the true increase in length produced by the operation was approximately $3 \mathrm{~cm}$.

\section{Vertebral body growth}

In the first 10 months after operation the length (corrected for magnification) along the margins of the vertebral bodies and intervertebral discs on the convexity of the curve in the fused area (distance $A$ in Fig. 2) increased by a mean $0.3 \mathrm{~cm}$ (range zero to $1.0 \mathrm{~cm}$ ). At final follow-up there was an additional mean increase of $1.6 \mathrm{~cm}$ (range 0.1 to $3.2 \mathrm{~cm}$ ) giving a total mean increase of $1.9 \mathrm{~cm}$ (range 0.5 to $3.7 \mathrm{~cm}$ ). The same measurements were made along the concave side of the vertebral bodies (distance $\mathrm{C}$ in Fig. 2), and during the first 10 months the mean increase was $0.2 \mathrm{~cm}$ (range zero to $0.6 \mathrm{~cm}$ ). By final follow-up there was a mean additional increase of $0.3 \mathrm{~cm}$ (range zero to $0.8 \mathrm{~cm}$ ), giving a total increase of $0.5 \mathrm{~cm}$ (range zero to $1.2 \mathrm{~cm}$ ). This increase is almost identical to the increase in the longitudinal axis (distance $\mathrm{D}$ in Fig. 2).

Post-operatively the sum of the heights of the vertebral bodies (distance B in Fig. 2) compared to the total length of the spine including the intervertebral discs 
in the fusion area (distance B plus disc spaces) was a mean of 82.3 per cent. After ten months this increased to a mean of 86.7 per cent, and at final follow-up it was 95.6 per cent. During the first ten months the mean sum of the heights of the vertebral bodies in the fusion area increased by $1 \mathrm{~cm}$ (range 0.4 to $1.8 \mathrm{~cm}$ ), and during the follow-up period there was a further increase of $2.2 \mathrm{~cm}$ (range 0.5 to $5.7 \mathrm{~cm}$ ), making a total of $3.2 \mathrm{~cm}$. The mean yearly
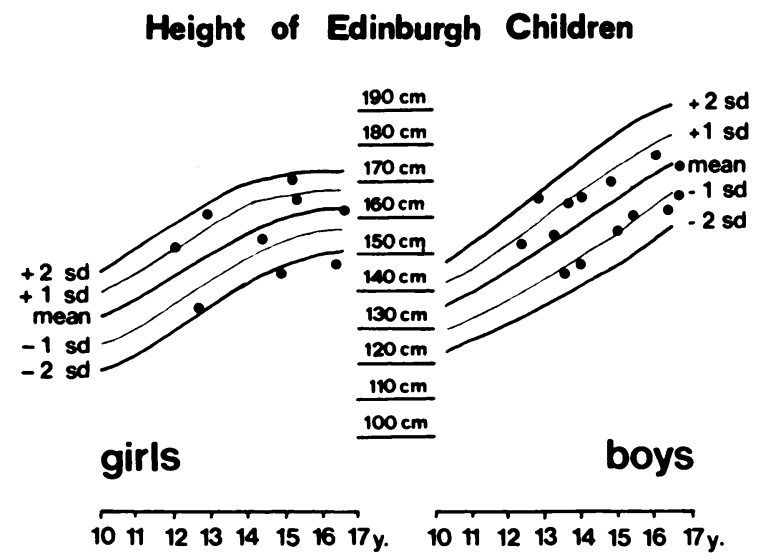

Fig. 5

Height of the 23 patients ( 9 girls and 14 boys) compared with the average height of Edinburgh children (standards of Department of Child Life and Health of the University of Edinburgh). The second standard deviation ( \pm 2 sd) corresponds approximately to the third percentile.

increase in the height of a vertebral body in the fusion area was, therefore, $0.067 \mathrm{~cm}$ and this was accommodated by narrowing of the intervertebral disc spaces. In two patients the quality of the radiographs did not allow accurate measurements and they were excluded.

The mean width of the apical vertebral body after operation was $32.8 \mathrm{~mm}$ (range 27 to $40 \mathrm{~mm}$ ) measured at its narrowest site, and $36.3 \mathrm{~mm}(32$ to $43 \mathrm{~mm}$ ) corrected for magnification on the radiograph at final follow-up. The mean increase in width of the vertebral body was, therefore, $3.5 \mathrm{~mm}$.

\section{Vertebral rotation}

The rotation accompanying the scoliosis was assessed by a modification of the method described by Nash and Moe (1969). In our method the rotation of the apical vertebral body was expressed as a percentage by comparing the distance between the border of the pedicle on the convex side and the border of the body on the same side to the total width of the vertebral body (Fig. 3). This percentage does not represent the actual number of degrees of rotation but can be used to show relative changes in rotation for a given patient when the measurements are made on the same vertebral body on different occasions. The mean rotation was 38.6 per cent (range 20 to 55 per cent) before operation, 33.5 per cent (range 18 to 43 per cent) after operation and 39.3 per cent (range 28 to 47 per cent) at final follow-up. These findings show that the correction of the scoliosis at the time of operation with Harrington instrumentation initially caused a partial derotation of the scoliosis, but during the following years the rotation again increased to its original severity and in some patients became more severe. The degree of initial derotation and subsequent rerotation varied considerably between the patients, but the tendency was always the same. In four patients the quality of the radiographs did not allow accurate measurements and they were excluded.

\section{Standing and sitting heights}

The standing heights of all but two of the patients at final follow-up were within the normal range for British children of an equivalent age (Tanner et al. 1966) (Fig. 5). Only two girls had standing heights below the third percentile and both these patients had small parents. The mean percentage of sitting to standing heights for all the patients was 50.8 per cent (range $\mathbf{4 2 . 5}$ to 53.8 per cent).

\section{Illustrative case}

A typical illustration case is shown in Figures 6-15.

\section{DISCUSSION}

A previous study from this Unit of children with idiopathic scoliosis fused posteriorly at 10 years of age showed that even a solidly fused spine continued to bend during the adolescent growth spurt, losing an average of 17 degrees of correction before becoming stable (McMaster and Macnicol 1979). Winter (1971) strongly advised supporting such young fusions with a Milwaukee brace to prevent bending during this period of rapid growth. Following these earlier findings it was decided by one of us to try to produce a more stable fusion by applying an additional massive onlay of autogenous iliac bone in an attempt to produce a wide and thick mass of bone which would be of sufficient strength to resist bending during the adolescent growth spurt. A single Harrington distraction rod used in combination with this fusion technique corrected the scoliosis by a mean of 51 per cent (mean 34 degrees), and all the spines developed a solid fusion. An underarm plaster jacket was worn for 10 months after operation while the fusion was becoming solid, and during this time there was a mean loss of correction of 4 degrees.

When the plaster jacket was removed the spine was left without support, and after a mean follow-up of 4.5 years there was an additional mean loss of 5 degrees despite the presence of the solid fusion. It is debatable whether this loss of correction is sufficiently severe to warrant the inconvenience of a Milwaukee brace, which would have have to be worn for several years after removal of the plaster jacket and might not be effective in stabilising the fusion.

The few previous studies of the growth of the spine after early posterior fusion have been based on the measurements of serial spinal radiographs (Ponseti and 
Friedman 1950; Risser et al. 1966; Winter 1971; Letts and Bobechko 1974). These studies suffer from the inaccuracy caused by varying radiographic magnificar overcome by calibrating our measurements against the length of the Harrington rod, which is a known constant on the serial radiographs for each patient. Our corrected

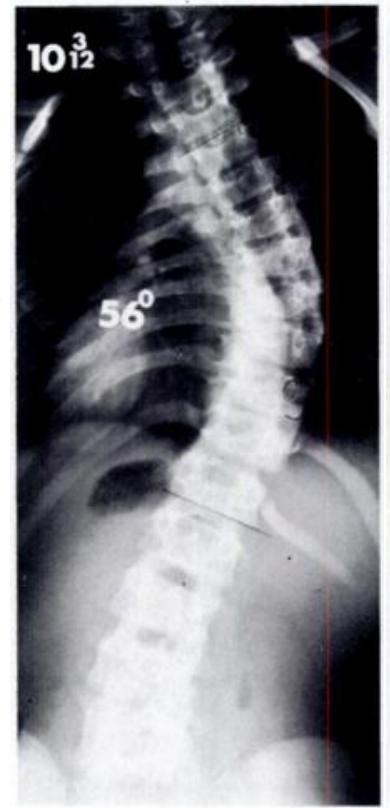

Fig. 6

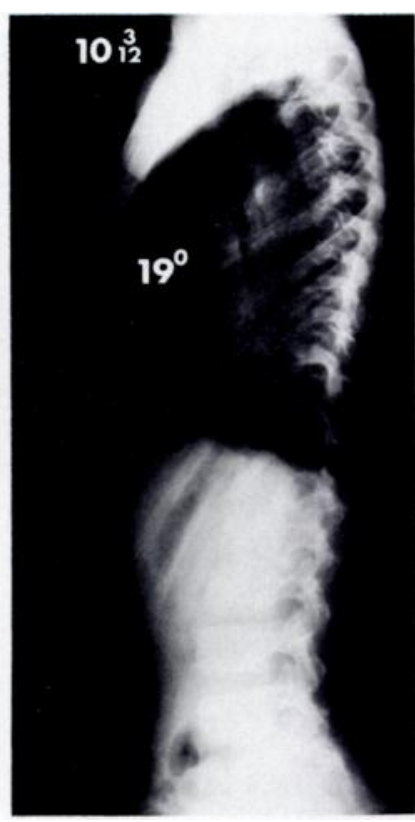

Fig. 7

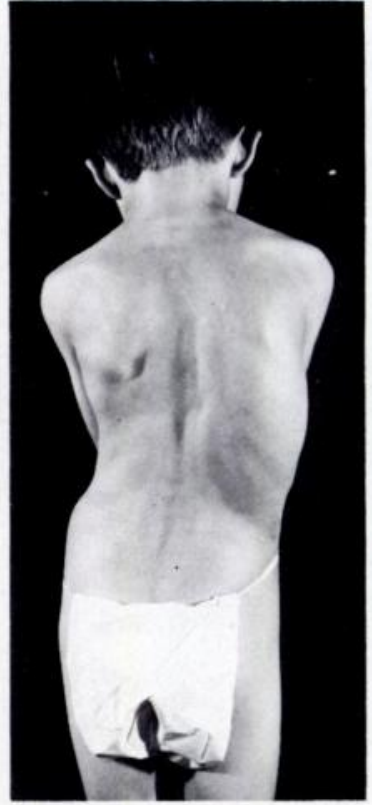

Fig. 8

Case 1. Figure 6-Boy aged 10 years 3 months with severe juvenile idiopathic right thoracic scoliosis. The pre-operative erect spinal radiograph shows the curve to measure 56 degrees. Figure 7 -Before operation there is a thoracic kyphosis of 19 degrees in the region of the proposed spinal fusion. Figure 8 -There is a marked rib hump on the right side.

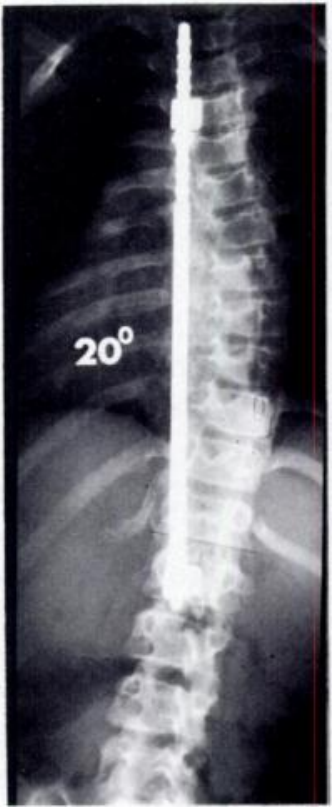

Fig. 9

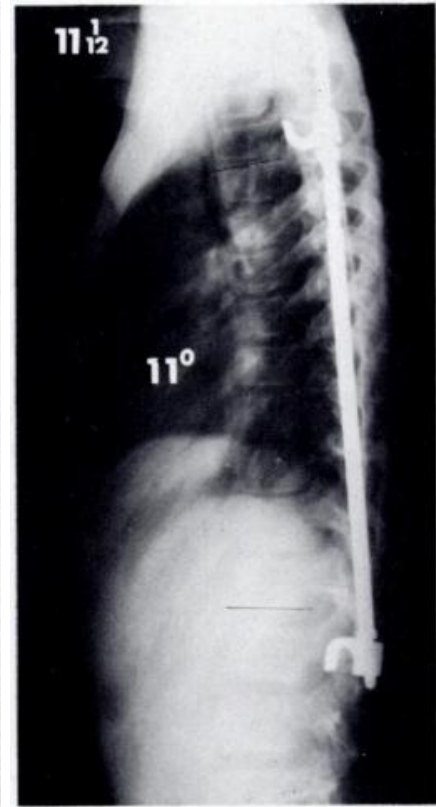

Fig. 10

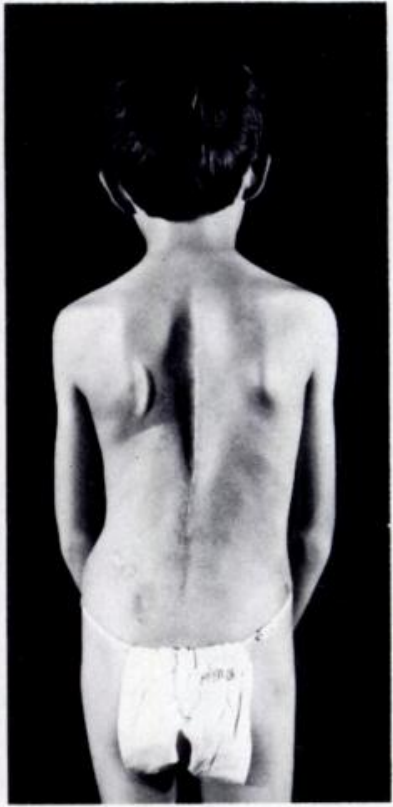

Fig. 11

Case 1. Figure 9-Ten months after posterior spinal fusion the radiograph shows good correction and solid fusion. The curve measures 20 degrees. Figure 10-After operation the kyphosis has been reduced to 19 degrees. Figure 11-Good cosmetic results; rib hump reduced.

tions of the spine produced by the differences in the distance of the patient from the radiographic plate on each occasion. In our study this inaccuracy has been serial measurements, taken over a mean period of four and a half years after solid fusion, showed no longitudinal growth in the posteriorly fused elements. This confirms 
the findings of Winter (1971) and of Letts and Bobechko (1974) but contradicts the observations of Risser et al. (1966).

Although the posterior fusion mass did not grow longitudinally, the vertebral bodies in the fused region continued to increase in height and width. The normal growth in height of one vertebral body is estimated to be $0.07 \mathrm{~cm}$ per year (Moe et al. 1978). In our patients, the growth in height of the vertebral bodies in the fused region was $0.067 \mathrm{~cm}$ per year and was therefore not significantly impaired by the fusion. This increase in height was presumably due to a continuation of the straighten the kyphosis as the lateral curvature was corrected (Fig. 10). After operation the thoracic kyphosis was reduced by a mean of 10.3 degrees, but on average there was no further reduction during the period of follow-up. Whereas most patients showed a mild further reduction of the kyphosis or remained unchanged, a few had an increase of kyphosis in spite of the growth of the anterior elements. We believe that the thick posterior fusion was of sufficient strength to prevent a significant reduction in the kyphosis or the development of a lordosis despite the tension produced by the continued longitudinal growth of the vertebral bodies anteriorly.

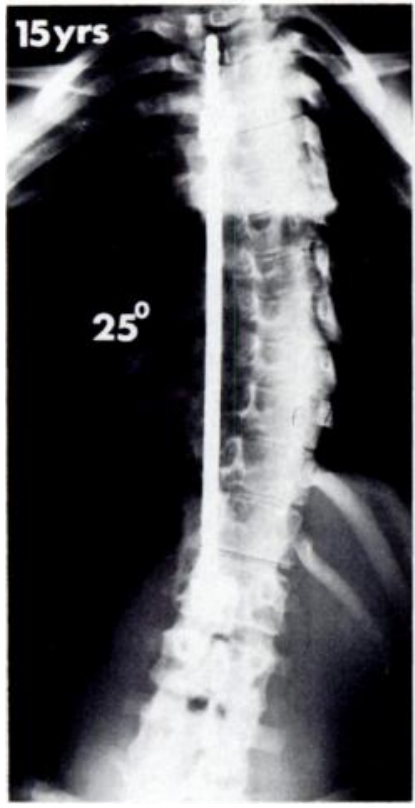

Fig. 12

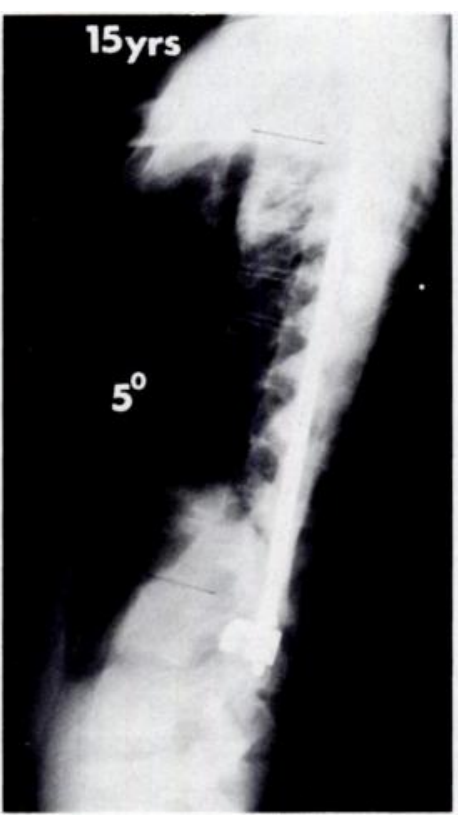

Fig. 13

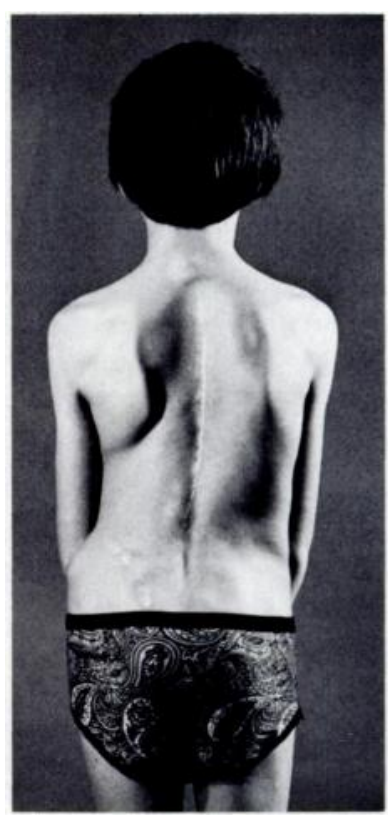

Fig. 14

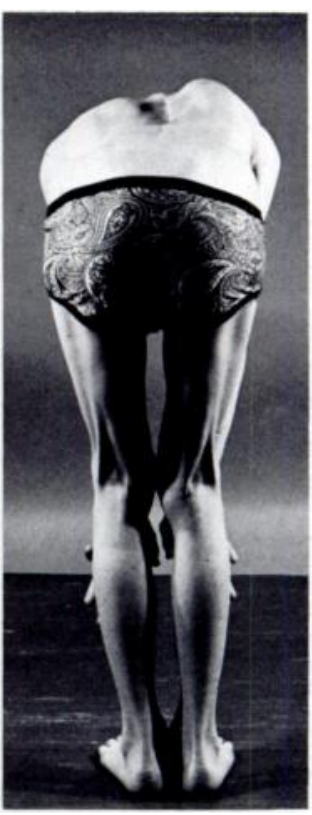

Fig. 15

Case 1. Figure 12-Radiograph taken at the age of 15 years shows that there has been a slight loss of correction and the curve now measures 25 degrees. The intervertebral disc spaces in the region of the spinal fusion are markedly narrowed and there has been a recurrence of the vertebral rotation. Figure 13 - The intervertebral disc spaces in the region of the fusion have narrowed considerably and there has been a slight loss in the post-operative degree of kyphosis. Figure 14-Cosmetically there is still a good result. Figure 15-The forward bending view shows that the rib hump has returned.

normal process of epiphysial growth at the end plates on the upper and lower surfaces of the vertebral bodies (Bick and Copel 1950). In our study the increase in height of the vertebral bodies in the posteriorly fused region was initially accommodated by narrowing of the intervertebral disc spaces - a finding previously noted by Risser $e t$ al. (1966). However, despite the narrowing of the intervertebral disc spaces there was no case of spontaneous interbody fusion. In animal experiments continued anterior growth of the vertebral bodies produced an increasing lordosis following posterior fusion (Veliskakis and Levine 1966; Coleman 1968). Bonnett et al. (1975) also noted a lordosis developing after long posterior fusions in young children with poliomyelitis. Before operation all of our patients had some degree of thoracic kyphosis, and four patients with infantile idiopathic scoliosis had a greater than normal kyphosis in the upper thoracic region. When the Harrington distraction rod was applied during operation it tended partially to
Our measurements have shown that during the follow-up period there was an increase of up to $2 \mathrm{~cm}$ in length of the convexity of the curve along the lateral margin of the vertebral bodies, with no corresponding increase on the concave side. It was also found that immediately after Harrington correction there was a partial reduction in the degree of vertebral rotation but that during the follow-up period the rotation again returned to the same severity or more. No measurements were made of the rib hump, but comparison of the clinical photographs taken before operation, when the plaster jacket was removed and at final follow-up showed that although the rib hump was reduced after operation (Fig. 11) it recurred as the patient grew (Fig. 15). In our opinion, the increase in length on the convexity of the curve and the recurrence of vertebral rotation are due to the continued longitudinal growth of the vertebral bodies. Once the degree of accommodation provided by the intervertebral disc spaces has been taken up, the vertebral 
bodies bulge laterally towards the convexity of the curve and pivot on the unyielding posterior fusion mass. It is likely that this contributes to the post-operative loss of correction and causes recurrence of the rib hump despite the presence of a solid posterior fusion. The increasing rib hump gives the illusion of a developing thoracic lordosis, but this is not confirmed on the lateral radiographs. So far as we know these observations have not been previously reported. These changes are not so apparent in patients with adolescent scoliosis, who are usually girls and undergo fusion after the age of thirteen years when the adolescent growth spurt is well advanced.

A criticism of early posterior spinal fusion is that because it prevents further longitudinal growth in the fused region it will produce a relative shortening of the spine as the child grows to maturity. Winter (1977) has produced a formula from standard growth tables which gives the approximate potential shortening of the spine caused by the fusion : centimetres of shortening $=0.07 \times$ number of segments fused $\times$ number of years of growth remaining. In our patients the mean number of segments fused was 11 , and assuming six years of growth remaining, the anticipated shortening caused by the spinal fusion would have been $4.6 \mathrm{~cm}$. It should, however, be remembered that the straightening of the spine at operation increased the overall height by approximately $3 \mathrm{~cm}$. Also if the spine had not been corrected and fused, the amount of shortening caused by the progressive curve is likely to have been greater than the shortening caused by the fusion. Although only six of our patients had reached skeletal maturity, the great majority had progressed sufficiently through the adolescent growth spurt to the stage where the growth rate was decelerating. At final follow-up only two of the 22 children were below the third percentile for the average height for British children of an equivalent age. However, on inspection the majority of children did appear to have mild to moderate stunting of the spine, and the mean percentage of sitting to standing height was 50.8 per cent, which is below the normal range of 52 to 53 per cent at skeletal maturity (Moe et al. 1978). This stunting of the spine is considered acceptable in comparison with the degree of deformity which would have occurred had the spine not been fused.

In conclusion we can state that although a posterior spinal fusion stops the longitudinal growth in the posterior elements, the vertebral bodies continue to grow anteriorly and this is most apparent during the adolescent growth spurt. A thick posterior fusion mass prevents the development of a lordosis but the anterior growth causes the vertebral bodies to bulge laterally towards the convexity and also to pivot on the posterior fusion, giving rise to a loss of correction, increasing vertebral rotation and recurrence of the rib hump.

\section{REFERENCES}

Bick EM, Copel JW. Longitudinal growth of the human vertebra: a contribution to human osteogeny. J Bone Joint Surg [Am] 1950;32-A: 803-14.

Bonnett C, Brown JC, Perry J, et al. Evolution of treatment of paralytic scoliosis at Rancho Los Amigos Hospital. J Bone Joint Surg [ Am] 1975; 57-A:206-15.

Coleman SS. The effect of posterior spine fusion on vertebral growth in dogs. J Bone Joint Surg [Am] 1968;50-A:879-96.

Harrington PR. Treatment of scoliosis: correction and internal fixation by spine instrumentation. J Bone Joint Surg [Am] 1962;44-A:591-610.

James JIP. Scoliosis. Edinburgh, London: E \& S Livingstone, 1967.

Letts RM, Bobechko WP. Fusion of the scoliotic spine in young children. Clin Orthop 1974; $101: 136-45$.

McMaster MJ, Macnicol MF. The management of progressive infantile idiopathic scoliosis. J Bone Joint Surg [Br] 1979;61-B:36-42.

McMaster MJ. Stability of the scoliotic spine after fusion. J Bone Joint Surg [Br] 1980;62-B: 59-64.

Moe JH. A critical analysis of the methods of fusion for scoliosis: an evaluation in two hundred and sixty-six patients. J Bone Joint Surg [Am] $1958 ; 40-A: 529-54$.

Moe JH, Sundberg B, Gustilo R. A clinical study of spine fusion in the growing child. J Bone Joint Surg [Br] 1964;46-B:784-5.

Moe JH, Winter RB, Bradford DS, Londstein JE. Scoliosis and other spinal deformities. Philadelphia, London, Toronto: WB Saunders, 1978: 74-80.

Nash CL, Moe JH. A study of vertebral rotation. J Bone Joint Surg [Am] 1969;51-A:223-9.

Ponseti IV, Friedman B. Changes in the scoliotic spine after fusion. J Bone Joint Surg [Am] 1950;32-A:751-66.

Risser JC, Norquist DM, Cockrell BR Jr, Tateiwa M, Hoppenfeld S. The effect of posterior spine fusion on the growing spine. Clin Orthop $1966 ; 46: 127-39$.

Tanner JM, Whitehouse RH, Takaishi M. Standards from birth to maturity for height, weight, height velocity and weight velocity: British children, 1965. Arch Dis Child 1966;41:454-71, 613-35.

Veliskakis K, Levine DB. Effects of posterior spine fusion on vertebral growth in dogs. J Bone Joint Surg [Am] 1966;48-A:1367-76.

Winter RB. The effects of early fusion on spine growth. In: Zorab PA, ed. Scoliosis and growth. Edinburgh, London: Churchill Livingstone, 1971 : 98-104.

Winter RB, Moe JH. The results of spinal arthrodesis for congenital spinal deformity in patients younger than five years old. $J$ Bone Joint Surg [Am] 1982;64-A:419-32.

Winter RW. Scoliosis and spinal growth. Orthop Rev 1977;6:17-20.

Zaoussis AL, James JIP. The iliac apophysis and the evolution of curves in scoliosis. J Bone Joint Surg [Br] 1958;40-B:442-53. 